\title{
TRABAJO INFORMAL: MOTIVOS, BIENESTAR SUBJETIVO, SALUD, Y FELICIDAD EN VENDEDORES AMBULANTES ${ }^{1}$
}

\author{
Emilio Moyano Díaz* \\ Ramón Castillo Guevara* \\ José Lizana Lizana*
}

\begin{abstract}
RESUMEN. Las relaciones entre trabajo, bienestar subjetivo (B.S.), felicidad y salud son complejas y de alto interés teórico y empírico. Ellas son analizadas aquí en trabajadores informales de comercio ambulante. Mediante observación y fotografías en las cinco ciudades más pobladas de la región del Maule (Chile), se registra 1556 puestos de venta de los que aleatoriamente se selecciona 258 para encuestar. Resultados: presentan una satisfacción vital global moderada, estando menos satisfechos con sus amigos y ocio, que con su trabajo y su familia. Su mayor felicidad proviene de ésta $\left(\chi_{\text {Friedman }}^{2}=389,47 ; g l=3 ; p<0,001\right)$. Están enfermos $M=6$ días mensualmente, aunque sólo dejan de trabajar un día al mes. Su B.S. correlaciona fuertemente con su satisfacción vital $(r=0,45)$, ésta con su felicidad $(r=0,44)$, y a su vez ésta con su salud $(r=0,42)$. Se discute éstos y otros resultados obtenidos.
\end{abstract}

Palabras-clave: Trabajo informal, bienestar subjetivo, salud

\section{TRABALHO INFORMAL: MOTIVOS, BEM-ESTAR SUBJETIVO, SAÚDE E FELICIDADE DE VENDEDORES AMBULANTES}

RESUMO. As relações entre trabalho, bem-estar subjetivo (B.S.), felicidade e saúde são complexas e de alto interesse teórico e empírico. Elas são analisadas aqui em trabalhadores informais do comércio ambulante. Mediante observação e fotografias nas cinco cidades mais populosas da região do Maule (Chile), registram-se 1556 postos de venda. Foram selecionados aleatoriamente 258 para interrogar. Resultados: os trabalhadores informais apresentam uma satisfação vital global moderada, estando menos satisfeitos com seus amigos e tempo de lazer do que com seu trabalho e sua família. Sua maior felicidade provém desta $\left(\chi_{\text {Friedman }}^{2} 389,47 ; g l=3 ; p<0,001\right)$. Encontram-se doentes $M=6$ dias mensalmente, ainda que só deixem de trabalhar um dia ao mês. Seu B.S. correlaciona-se fortemente com sua satisfação vital $(r=0,45)$, esta com sua felicidade $(r=0,44)$ e esta última com sua saúde $(r=0,42)$. Estes e outros resultados sãodiscutidos neste artigo.

Palavras-chave: trabalho informal, bem-estar subjetivo, saúde.

\section{INFORMAL WORK: MOTIVES, WELL BEING, HEALTH AND HAPPINESS IN STREET VENDORS}

\begin{abstract}
Relationships among work, subjective well being (SWB), happiness and health are complex and have a high theoretical and empirical relevance. In this study, these relationships are analyzed in street vendors. Using observation and photographs in the five bigger cities Maule Region (Chile), 1556 sale points were found and 258 of them were randomly selected for this study. Results: vendors have a moderated life satisfaction, with lower satisfaction levels about their friends and leisure than about their work and family. The highest report of happiness comes from their family $\left(\chi_{\text {Friedman }}^{2}=389,47 ; g l=3\right.$; $p<0,001)$. They report being sick $M=6$ days per month, although they only miss work one day per month. Their SWB
\end{abstract}

\footnotetext{
El presente estudio forma parte del Programa de Investigación “Calidad de Vida y Ambientes Saludables” (Res. 387/2007) de la Facultad de Psicología de la Universidad de Talca.

* Facultad de Psicología, Universidad de Talca, Talca, Chile.
} 
correlates with their life satisfaction $(r=0,45)$, happiness $(r=0,44)$, and health level $(r=0,42)$. These and others results are discussed in the study.

Key words: Informal work, well being, health.

El crecimiento económico chileno en 2006 fue de $4 \%$, inferior al registrado en $2005(5,7 \%)$ y 2004 (6,2\%). Sin embargo, el porcentaje de desempleados descendió en 2006 a un 7,8\% de la población económicamente activa, contra un $9.2 \%$ de 2005 y 10\% de 2004 (Banco Central de Chile, 2007). La controversia de 2004 respecto de si eso significaba que se estaba con una economía en crecimiento sin empleo se ha cambiado por otra que interpela por qué no ha habido un crecimiento mayor.

La incorporación de Chile a la Organización para el Desarrollo y Desarrollo Económico (OCDE) mejora el estándar de calidad del método para el cálculo del empleo. En la metodología "antigua" -1960- para ello, propia del Instituto Nacional de Estadísticas y de la Universidad de Chile, se considera 'empleados' también a los trabajadores 'por cuenta propia' o sin contrato o, más generalmente, 'informales'. Así, cuando éstos dejan de laborar el desempleo aumenta o, cuando se incorporan más personas a este trabajo 'informal' el desempleo general disminuye. Esto hace difícil la interpretación de las cifras de empleodesempleo y muy especialmente las conjeturas acerca de las motivaciones de los trabajadores asalariados por eventualmente buscar -contraintuitivamente- el trabajo informal ó, más convencionalmente, a la inversa. Una hipótesis respecto al lo primero podría ser que la calidad del trabajo asalariado o formal es mala lo que llevaría a algunos trabajadores a emprender su propio negocio - generalmente informal en un primer momento - mientras otros, provenientes del trabajo informal y no suficientemente satisfechos con él, busquen la 'seguridad' del trabajo asalariado.

El concepto de 'economía informal', de mayor complejidad que el de 'sector informal', se refiere al grupo cada vez mayor y diverso de trabajadores y empresas, (urbanas o rurales), que operan en el ámbito informal. Incluye trabajadores por cuenta propia dedicados a actividades de subsistencia, tales que vendedores callejeros, lustrabotas, traperos, chatarreros, recogedores y recicladores de basura, trabajadores a domicilio, y los pertenecientes a empresas o fábricas en que se les hace pasar por asalariados, trabajadores independientes de microempresas que funcionan aisladas con trabajadores familiares $\mathrm{o}$, a veces, con aprendices (OIT, Organización Internacional del Trabajo, 2002, p. 2-3). Estos trabajos no están reconocidos ni protegidos jurídica ni reglamentariamente englobando a trabajadores de alta vulnerabilidad a quienes a veces se les confunde con delincuentes siendo objeto de prejuicio, discriminación y represión, no obstante tratarse en su gran mayoría de trabajadores que producen bienes o servicios legales (OIT, 2002, p. 3).

La economía informal en el mundo ha crecido y tiene gran potencial de seguir creciendo, a causa del desempleo de alrededor de 160 millones de personas (de los cuales 53 millones en países industrializados y en desarrollo) a los que se agrega 310 millones de personas más, para quienes no hay ocupaciones suficientes. La economía global ha crecido en 40 millones de puestos de trabajo por año mientras habría 48 millones de personas buscando empleo (OIT, 2002, p. 137). La mayoría de las personas que no encuentran empleo en el sector formal recurren entonces al sector informal de empleos independientes y por cuenta propia.

Tanta importancia tiene el trabajo informal en América Latina que hay quienes estiman que la economía de dos potencias económicas regionales México y Brasil- no podría ser adecuadamente comprendida sin hacer referencia a aquel. En otros países como por ejemplo Perú, se estima que el $42 \%$ de su Producto Nacional Bruto (PNB) es el resultado del trabajo informal, mientras que en Argentina el $70 \%$ del PNB corresponde a economía informal y el $50 \%$ de las horas hombre trabajadas en la oficina son ilegales (de Soto, 1987). Otro estudio indica que en Argentina el $20 \%$ de los trabajadores informales es subcontratado para proyectos gubernamentales $\mathrm{y}$, en Puerto Rico, se estima que el 23,3\% del PIB proviene de la economía informal (Ocasio, 2005; Market: Latin America, 2004).

Los motivos para explicar la fuerte incidencia del trabajo informal sobre el PIB de éstos y otros países, probablemente puedan ser explicados por la economía. En el presente estudio en cambio interesan las razones y motivos para realizar dicha actividad dados desde la subjetividad $\mathrm{y}$ vida de sus actores mismos o participantes directos.

\section{EL TRABAJADOR Y LA MOTIVACIÓN AL TRABAJO}

La psicología enseña que es más probable que los individuos trabajen después de períodos de ocio, 
descanso o aburrimiento, y que cesen de hacerlo para procurarse ocio, descanso o aburrimiento, y así sucesivamente. El trabajo brinda la posibilidad de experimentar los desafíos y alegrías del trabajo bien hecho, procurar la virtud y la alegría y, tal vez, la escurridiza felicidad en ello.

La mayor parte de los seres humanos trabaja porque necesita hacerlo incluso quienes no lo hacen por dinero. Necesidad y deseo se imbrican; hoy se necesita trabajar para poder obtener ingresos que permitan vivir (ayer para obtener alimento), pero el trabajo es una fuente no sólo de ingresos sino también de puesta en práctica de capacidades y habilidades personales, de enfrentar desafíos y así, ni más ni menos, de la propia realización personal. A quienes les gusta su trabajo no lo dejarían aún cuando no necesitaren el dinero que les procura (Argyle, 1992).

El trabajo sentido como una carga es más probable cuando quien lo ejerce lo hace instrumental o exclusivamente para obtener un ingreso. Argyle (1992), ha señalado que el trabajo aporta menos a la felicidad total de las personas comparativamente que la pareja, la familia, el ocio o los amigos, pero, por el contrario, tiene un potencial de infelicidad altísimo y demostrado.

La teoría del flujo (Csikszentmihalyi, 1999) postula que el bienestar psicológico (B.P.) radicaría en la actividad humana en sí y no en la satisfacción o logro de la meta final. La actividad laboral tiene el potencial de producir un sentimiento especial de flujo. Así, una aspiración legítima y con cierta base psicológica para las personas es la búsqueda de un trabajo que les provea experiencias de flujo. La expresión 'pasatiempo remunerado', siendo una contradicción de los términos, podría transmitir quizá adecuadamente esta condición del trabajo como una principal fuente de entretención, de prueba exitosa de habilidades personales y, así, de bienestar psicológico, satisfacción vital y eventualmente de felicidad.

El B.P. es un componente del concepto mayor de bienestar subjetivo y a su vez éste hace parte de un universo aún mayor -definido por distintas disciplinas- como es el de calidad de vida. Son dos las aproximaciones generales y una tercera de síntesis dialéctica, para aproximarse al B.P. Por una parte, hay quienes asumen que éste sería una característica (rasgo) asociada a la personalidad y por lo tanto, bastante estable en el tiempo y poco afectada por los acontecimientos de la vida. A esta asunción de rasgo se le ha denominado teoría top-down (de arriba a abajo) mientras hay quienes postulan una teoría alternativa bottom up (de abajo hacia arriba) donde se asume que experiencias de bienestar específicas (estados) respecto de diferentes ámbitos del quehacer humano (familia, trabajo, etc.), van generando en las personas un sentimiento más global o generalizado de bienestar. Así, la satisfacción vital sería el resultado de tener muchos momentos felices en los diferentes dominios vitales (Díaz \& Sánchez-López, 2002).

Ambos enfoques provienen evidencia a su respectivo favor lo que ha llevado a la formulación de un tercer enfoque, en el que se asume que las influencias entre estado ó rasgo respecto del ambiente son bidireccionales de modo tal que rasgos (positivos) de interpretación de la realidad llevan más probablemente a extraer satisfacción de los eventos de la vida, y que la sumatoria de éstos a su vez contribuyen a crear satisfacción vital de modo generalizado.

Dentro de este mapa conceptual se inscribe el concepto de satisfacción vital, el que ha sido concebido como el componente cognitivo del bienestar subjetivo, y también a su vez, pudiendo enfocarse como rasgo o como estado. Otro concepto asociado y mayor por su amplitud o extensión es el de felicidad, el que también puede plantearse como rasgo o como estado, y que para algunos incluiría el bienestar subjetivo y la satisfacción vital (S.V.), mientras que para otros sería solo el componente afectivo ó emocional de éste último (Moyano Díaz, 2004a).

Diener (1984) y Myers (1993) han revisado reportes a gran escala realizados en USA que muestran que la gente es más feliz de lo que uno podía suponer y que ello no parece depender de modo significativo de las circunstancias externas. La proporción de quienes declaran ser 'muy felices' ha declinado de $35 \%$ al $29 \%$ de 1957 a 1996 en USA, no obstante ganan dos veces más dinero que antes (Myers \& Diener, 1997). El dinero parece ser eficiente para incrementar la felicidad de quienes son muy pobres pero su impacto disminuye o se anula para quienes no están en esa condición. Para estos últimos se ha propuesto investigar la salud mental, el trabajo satisfactorio y seguro, la vida privada y amorosa, la comunidad segura, la libertad y los valores morales (Layard, 2003, p. 27).

Ha sido reportado un cierto número de teorías acerca de las causas de la felicidad, entre las que están: la referida a objetivos, sugiriendo que la felicidad se logra cuando algún estado, meta o necesidad es satisfecha, la de la actividad, que sugiere que la felicidad puede ser lograda a través de la interacción social o del ocio, y la de comparación social postulando que la felicidad resulta de una 
comparación entre un estándar y una condición actual (Furnham \& Chang, 2000).

Quienes trabajan principalmente por obtener un ingreso dejarían de hacerlo si ganasen la lotería o se volvieran ricos súbitamente, mientras no ocurre así con quienes sienten que su trabajo es una oportunidad de realización y ejercicio de motivaciones profundas, los que continuarían trabajando aún sin necesidad económica de hacerlo (Argyle, 1992). Hay un número no menor de personas en el mundo ejerciendo un trabajo voluntario (vocacional probablemente), sin recibir remuneración, para quienes el reconocimiento y la aprobación sociales, probarse en habilidades para superar desafíos, dignidad, pasar el tiempo o combatir el aburrimiento o los problemas familiares, la búsqueda de emociones y experiencias nuevas, conocer y ayudar a otros, entre otros, son motivos suficientemente poderosos.

Quienes tienen menor calificación laboral o educación sistemática, presentan como principal motivación para decidir por un empleo u otro el monto de la remuneración, mientras que quienes han tenido una educación o capacitación más prolongada tienen como principal motivación para ello si el empleo en cuestión les provee o no de oportunidades para ejercer sus aptitudes en lo que les gusta hacer (Argyle, 1992). Evidentemente, es un ideal socialmente muy deseable para la sociedad en su conjunto que su población económicamente activa se desempeñe laboralmente en la condición que hemos denominado 'vocacional' ya que por una parte, trabajadores felices rinden más ó, al menos, ello sería un factor protector de conflictos, accidentes, robos, sabotaje y de mal clima laboral.

Por cierto, aún lejos del ideal de proveer trabajo 'vocacional' o de alta calidad, parece imprescindible al menos - exigir un 'trabajo decente', definido por la (OIT, 1999) como aquel realizado bajo condiciones humanas de seguridad físico-ambiental, protección bajo enfermedad, previsión, derecho al descanso, etcétera. Así, y especialmente en un país que puede autoevaluarse positivamente respecto de su crecimiento económico, esto lleva a la pregunta por la calidad del trabajo que le caracteriza, ya que ello es constitutivo de la calidad de vida de su población y es un analizador natural del grado de bienestar de la misma.

\section{TRABAJO INFORMAL Y CALIDAD DEL TRABAJO}

La calidad del empleo ha sido definida a partir de tres dimensiones; regularidad, protección y autonomía (Touraine, 1998), las que parecen ser apropiadas al menos para el análisis de la calidad del trabajo de tipo formal. La definición de calidad del trabajo puede diferir entre regiones y naciones, además de entre individuos, y a su vez en éstos variar en el tiempo según su edad o etapa de vida en que estén (Carty, 1993). La O.I.T. define un empleo de calidad como aquel que es seguro, estable, libre de riesgo para la salud física y mental en que se desempeña el trabajador, realizado bajo condiciones de dignidad y respeto a la seguridad y leyes sociales del trabajo (OIT, 1999). Dadas las condiciones de precariedad, inseguridad, insalubridad y otras condiciones negativas del trabajo en el mundo - mala calidad del empleo -, la OIT (1999) ha definido entre sus temas de orientación doctrinaria y de política prioritaria el 'trabajo decente'. Este concepto alude a trabajo seguro, con respeto a las leyes sociales y protectoras del trabajador y su integridad física y psicológica, y es instituido como prioridad de la OIT desde 2000 para su promoción y respeto en los países del mundo.

Un informe que resume parte del debate sobre la calidad del empleo en Estados Unidos de América de 1970 a 1990, reporta que hay un incrementado número de desertores en buscar empleo, especialmente entre los más jóvenes, lo que es indicativo de la existencia de muchos trabajos de bajo salario que no proveen suficiente incentivo para trabajar, “...o dónde los incentivos ofrecidos por el mercado ilegal son más lucrativos" (Carty, 1993, p. 25). Sin duda este es el caso del comercio ilegal de drogas, o de música, o de DVDs con películas "pirateadas" en numerosos países del mundo. En este contexto, sólo los jóvenes que disponen de la educación y destrezas que específicamente pide el mercado tienen empleo.

En América Latina el porcentaje de empleo informal urbano con respecto al empleo total urbano creció del $50 \%$ en 1990 al $58 \%$ en 1997 , atribuido a aumento de la mano de obra a causa de factores demográficos, de un ascenso del índice de actividad, particularmente de la mujer - y a la inmigración rural urbana, además de una reducción del empleo en el sector formal. La precariedad laboral persiste, ya que sólo 4 de 10 nuevos ocupados tiene acceso a los servicios de la seguridad social, y sólo 2 de cada 10 ocupados en el sector informal cuentan con protección social entre 1990 y 2002 (OIT, 2003). La informalización del empleo en América Latina y El Caribe se evidencia en el hecho que de cada 10 nuevos ocupados de 1990 a 2002, 7 han sido informales (OIT, 2003). La estimación del número de personas que se desempeña laboralmente en lo informal está sujeta a altísimo error. Se trata de personas que por motivos variados tales que falta de educación y capacitación, 
de oportunidades, de segregación social racial o provocada por limitaciones físicas, o tal vez por la sencilla razón de que disponen de un empleo 'formal' mal remunerado, buscan aumentar su ingreso por la vía informal (Yunus, 2000). Algunas de las hipótesis relativas a los motivos para dedicarse al trabajo informal que serán sometidas a verificación aquí son: no encontrar trabajo asalariado, discriminación, abandono de algún trabajo asalariado anterior, incrementar el dinero proveniente de un trabajo asalariado actual (Yunus, 2000).

Otras motivaciones para desempeñarse en el trabajo informal son la falta de oportunidades en el área de especialización o de estudio, la falta de confianza en las instituciones gubernamentales o públicas, el excesivo número de trámites para formalizar el trabajo cuando este tiene la posibilidad de ser legalizado (de Soto, 1987) o la alternativa del "dinero fácil", como es el caso de los traficantes de drogas (Ocasio, 2005).

Existen diversos métodos de evaluación de la calidad del empleo, y entre los criterios utilizados para ello están (Touraine, 1998): a) Ingreso y beneficios similares, b) Horas de trabajo, su organización y la intensidad del trabajo, c) La seguridad, el sentido de estabilidad y la confiabilidad del empleo, d) Los riesgos de accidentes, los peligros ocupacionales para la salud, e) El ambiente del trabajo (ruido espacio, interacción social), f) La protección social, caso de enfermedad, despido o jubilación, g) El interés contenido y las oportunidades que ofrece el empleo para el desarrollo personal, h) El contexto ético y moral, el valor y estatus social proporcionado por el empleo e i) la participación en la determinación de las condiciones del trabajo.

Generalmente las investigaciones sobre la calidad del trabajo incluyen sólo las dimensiones a y f, más fácilmente operacionalizables, y una visión integrada del conjunto de factores no parece disponible aún. Es razonable asumir que algunos tipos de trabajo, como el trabajo asalariado no protegido, el de temporero, y el trabajo no profesional por cuenta propia (informal), tienen menos niveles de calidad que los trabajos asalariados protegidos con contrato permanente. Interesa explorar aquí las características de este tipo de trabajo por cuenta propia o también denominado "informal", realizado por no profesionales y particularmente por comerciantes ambulantes en la región del Maule, Chile.

Se medirá aquí la calidad de este tipo de trabajo informal identificando las explicaciones que entregan de las eventuales ventajas de su trabajo informal respecto del trabajo formal, y objetivando la evaluación que los trabajadores mismos hacen de su
B.P. en él, de su S.V., y de cuánto les aporta a su felicidad respecto de lo que les provee la familia, las amistades, o el ocio. Un aspecto adicional evaluado aquí es el de la satisfacción laboral (S.L.), tema extensivamente desarrollado respecto al trabajo formal pero cuyo análisis y reporte es más bien escaso en trabajadores informales.

\section{METODO}

\section{Muestra}

De un total de 1556 puestos de trabajo callejero observados en cinco ciudades de la región del Maule ${ }^{2}$, en Chile, se extrajo aleatoriamente una muestra proporcional de 258 trabajadores, con un nivel de confianza superior al $95 \%$, in situ, mediante un procedimiento en zig-zag, asegurando cubrir todo el espacio urbano ocupado, en un número calculado según el tamaño de cada lugar (medido en número de puestos de trabajo) y calculando un coeficiente de elevación para cada lugar.

\section{Instrumentos}

Fue elaborada una "Escala de Bienestar General" (EBG) consistente en cuatro instrumentos: escala de bienestar psicológico, escala de salud adaptada de la CDC's Health-Related Quality of Life (Hennessy, Moriarty, Zack, Scherr, Brackbill, 1994; Hagerty et al., 2001), escala de satisfacción vital (de Diener, Emmons, Larsen y Griffin, citados por Seligman, 2003), y escala de felicidad (de Lyubomirsky, S. \& Lepper, H. S., citados por Hagerty et al., 2001). La confiabilidad obtenida (Cronbach) en población universitaria $(N=255)$ para la escala de satisfacción vital es .82 y para la de felicidad general .76. (Moyano Díaz, 2004b).

\section{Procedimiento}

Los instrumentos a la muestra definitiva fueron aplicados in situ por tres estudiantes avanzados de psicología entrenados ad-hoc, en horas de trabajo

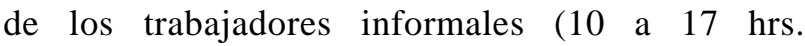
modalmente).

\section{RESULTADOS}

Los resultados son resumidos en tres acápites: descripción sociodemográfica, verificación de

2 Las ciudades de Talca, Curicó, Linares, Cauquenes, y Constitución. 
hipótesis de motivos para trabajar informalmente $\mathrm{y}$, evaluación de las variables psicológicas (B.P., S.V., Felicidad) medidas en los trabajadores informales.

\section{Descripción sociodemográfica}

La muestra está casi equirepartida por sexo, 128 mujeres y 130 hombres, con una edad promedio de 45 años $(D E=11,78)$, en rango de 20 a 78 años. El $70 \%$ se declara casado(a), el $19 \%$ soltero(a), el $6.2 \%$ separado(a), el 4,7\% viudo(a) y 1 divorciado(a). Casi un $70 \%$ declara tener casa propia, y un $15,5 \%$ y un $12 \%$ vivir en arriendo y de allegados respectivamente. El $77 \%$ ha desempeñado otro trabajo antes, la inmensa mayoría se autodefine como comerciante, más escasamente hay lustrabotas y sólo uno se dedica a fletes. Se incluye entre ellos a vendedores de comestibles, de ropa (calcetines, pinzas, ropa interior, entre otros), accesorios de celulares, de frutas y verduras, feriantes, etc. Trabajan modalmente 7 días por semana (rango de 1 a 7 días), y su antigüedad promedio en la actividad es de casi 13 años, y 20 años la moda en un rango que va de 6 meses a 50 años. Para el $80 \%$ de la muestra el ingreso promedio diario es de $\$ 8.900$, aunque hay un $7 \%$ que declara ganar más de $\$ 16.500$ pesos diarios (U\$35, 1 U\$ $=471$ pesos chilenos a enero de 2008).

\section{Verificación de hipótesis: motivos reportados para devenir comerciante ambulante}

Aquí se somete a verificación cuatro hipótesis respecto a los motivos por los cuales los vendedores ambulantes se dedican a esta actividad. El análisis estadístico efectuado es bajo el supuesto de igualdad en la proporción de respuestas negativas y positivas a la pregunta formulada en cada caso.

Casi dos tercios de los trabajadores declaran dedicarse a este trabajo porque en su momento no pudieron encontrar un trabajo asalariado $\left(\chi^{2}=10,93\right.$; $p<0.01)$, y que la causa de esto no ha sido la discriminación $\left(\chi^{2}=15,02 ; \mathrm{p}<0.05\right)$. No obstante esto, existe más de un tercio de trabajadores(as) quienes indican como causa haber sido objetos de discriminación. Casi otro tercio declara haber pasado un largo período de cesantía, un $17 \%$ considera haberse dedicado a este trabajo porque aparece como un negocio atractivo, y en el cual se obtiene dinero diariamente $(16 \%)$, o porque se trata de un negocio familiar (14\%), o porque es lo único que sabe hacer $(8 \%)$.

La hipótesis relativa a que los trabajadores voluntariamente han dejado un empleo formal para iniciar su propio negocio aunque no es estadísticamente corroborada $\left(\chi^{2}=0,14\right.$; n.s. $)$ aparece como relevante ya que la mitad de estas personas señala haber dejado sus trabajos asalariados como iniciativa libremente elegida para dedicarse a esta actividad, mientras la otra mitad se vio obligada a hacerlo.

Respecto de si la realización del trabajo informal ocurre en paralelo con otro trabajo formal o asalariado cuyo ingreso es insuficiente, sólo un $14 \%$ de la muestra estaría en esa condición, siendo significativa la gran mayoría para quienes su trabajo como comerciante informal es su único trabajo y fuente de ingreso $\left(\chi^{2}=129,39 ; p<0.01\right)$.

En el trabajo informal la libertad de decidir cuándo y en qué horario trabajar es un motivo de ventaja significativa al compararlo con el trabajo formal $\left(\chi^{2}=415,72 ; p<0.01\right)$.

\section{Descripción de dimensiones psicológicas de los trabajadores informales}

B.P., salud, creencias acerca de las que serían principales fuentes de felicidad, S. V. y felicidad de los vendedores ambulantes.

\section{Bienestar Psicológico}

El actual nivel de satisfacción en cada uno de los ámbitos de participación social analizados muestra que éste aparece global y positivamente moderado y va de 2,57 (sobre 5) como valor más bajo (Amigos, D.E. = $1,36)$ a 4.73 como el más alto (Familia, D.E.= 0,57). También aquí se observa que la distribución de estas valoraciones son estadísticamente diferentes, dónde la satisfacción respecto de la familia y el trabajo es significativamente superior a la relativa al ocio o descanso y, muy por encima de la relativa a los amigos $\left(\chi_{\text {Friedman }}^{2}=389,47 ; g l=3 ; p<0,001\right)$. Adicionalmente, la variabilidad en las respuestas relativas a la satisfacción con la familia es la más baja mientras la relativa a los amigos es la más alta.

\section{Salud}

Condición básica para que las personas puedan trabajar es la salud, y el trabajo informal de vendedor ambulante presenta como componente central de su definición su inseguridad y falta de protección, estando eventualmente expuesto a un ambiente de trabajo desprotegido e insalubre. Cuando el (la) trabajador(a) enferma no tiene seguridad social ni atención sanitaria y dejará de obtener dinero durante sus días no laborados. Los resultados (Tabla 1) muestran que estos trabajadores presentan una media de casi 6 días al mes físicamente enfermos, con una desviación estándar de 8.9 días. Con una dispersión semejante 
reportan un promedio de casi 5 días al mes psicológicamente enfermos, y casi tres días en que tanto su salud física como su salud mental están negativamente afectadas. Sin embargo, reportan que sólo un día y medio se abstuvieron de ir a trabajar, lo que puede ser considerado ilustración adicional de la precariedad de su trabajo.

Tabla 1. Escala de Bienestar en Salud $(N=258)$.

\begin{tabular}{lcc}
\hline Reactivos & Media & d.e. \\
\hline $\begin{array}{l}\text { Días durante los últimos } 30 \text { días, en que su salud } \\
\text { física no buena. }\end{array}$ & 5,64 & 8,96 \\
$\begin{array}{l}\text { Días durante los últimos } 30 \text { de salud mental no buena } \\
\begin{array}{l}\text { Días durante los últimos } 30 \text { de salud física y mental } \\
\text { simultáneamente no buenas. }\end{array}\end{array}$ & 2,71 & 6,74 \\
$\begin{array}{l}\text { Días en que una mala salud física o mental le abstuvo } \\
\text { o impidió de realizar sus actividades habituales } \\
\text { (cuidado personal, trabajo, estudio o recreación). }\end{array}$ & & 4,21 \\
\hline
\end{tabular}

Legenda: Desviación estándar (d.e.)

\section{Creencias acerca de las fuentes de felicidad}

Estos trabajadores creen que la principal fuente de felicidad de las personas es su familia, seguida por el trabajo, el descanso u ocio y, finalmente los amigos $\left(\chi^{2}\right.$ Friedman $\left.=422,97 ; g l=3 ; p<0,001\right)$. La familia es también el subsistema acerca del cual hay mayor consenso, es decir, la importancia atribuida a la misma como fuente de felicidad es la que presenta menor dispersión en la muestra. Las diferencias entre las creencias acerca de las fuentes de felicidad es significativa, es decir, claramente estas fuentes de felicidad son diferentes respecto de su lugar ocupado en una jerarquía de fuentes de felicidad, como se registra en la Tabla 2.

Tabla 2. Creencias Acerca de las Fuentes de Felicidad.

\begin{tabular}{lcc}
\hline Reactivos & Media* & d.e. \\
\hline $\begin{array}{l}\text { El trabajo para las personas es su principal } \\
\text { fuente de felicidad }\end{array}$ & 3,73 & 1,27 \\
$\begin{array}{l}\text { La familia es para las personas su principal } \\
\text { fuente de felicidad }\end{array}$ & 4,79 & 0,52 \\
$\begin{array}{l}\text { Los(as) amigos(as) son la principal fuente de } \\
\text { felicidad para las personas }\end{array}$ & 2,05 & 1,13 \\
$\begin{array}{l}\text { El descanso (ocio) es lo que produce la mejor } \\
\text { felicidad en las personas }\end{array}$ & 2,93 & 1,51 \\
\hline
\end{tabular}

Legenda: Desviación estándar (d.e.)

* 1= muy en desacuerdo; $7=$ muy de acuerdo

\section{Felicidad y variables sociodemográficas}

No se observan diferencias significativas respecto de la felicidad entre hombres (Media $=5,28$; $D E=0,98)$ y mujeres (Media $=5,01 ; D E=1,31)(t=1,86$; $g l=253 ; p=0,065)$. Tampoco se observa relación entre la edad y la felicidad $(r=-0,12 ; p=0,064)$. Tampoco se registra diferencia significativa según estado civil de casados (Media $=5,21 ; D E=1,47)$ respecto de otros estados civiles (Media $=4,99 ; D E=1,20)(t=1,29 ; g l$ $252 ; p=0,19)$.

\section{Satisfacción Vital}

No se registra diferencias significativas en la S.V. entre hombres (Media $=3,55 ; D E=0,95)$ y mujeres (Media $=3,36 ; D E=0,98)(t=1,52 ; g l=248 ; p>0,1)$. Siguiendo la tendencia observada en el análisis relativo a la felicidad, la relación entre la edad y satisfacción es nula ( $r=-0,03 ; p>0,1)$. Respecto de la variable estado civil se observa que los casados (Media $=3,57 ; \quad D E=0,89$ ) experimentan mayores niveles de S.V. que los de otros tipos de estado civil (Media=3,21; $D E=1,09)(t=2,81 ; g l=247 ; p<0,01)$.

\section{Algunas relaciones entre las dimensiones psicológicas evaluadas}

La matriz de correlaciones muestra que las variables se encuentran relacionadas entre sí. Es destacable que el constructo B.P. correlaciona fuertemente con la satisfacción vital $(r=0,45 ; p<0,01)$, ésta con la felicidad $(r=0,44 ; p<0,01)$ y a su vez ésta con la salud $(r=0,42 ; p<0,01)$. Las correlaciones entre felicidad y B. P. $(r=0,29 ; p<0,01)$, de éste con salud $(r=0,18 ; p<0,01)$ y de ésta con la satisfacción vital $(r=$ ,28; $p<0,01)$ son más bien débiles.

Pareciera existir una gran dimensión de bienestar general, sin embargo en el detalle es posible observar que el constructo B.P. está fuertemente correlacionado con la satisfacción vital (evaluación general de la vida llevada hasta el momento de la evaluación), sugiriendo una mutua retroalimentación. Así, quien no cambiaría nada de su vida si tuviera la posibilidad de vivirla nuevamente está a la vez satisfecho con su vida en áreas más específicas de participación social tales que trabajo, familia, amigos y ocio. A su vez quien está satisfecho globalmente con lo que ha sido su vida se declara más probablemente feliz, con un mayor nivel de felicidad. El nivel de felicidad experimentada a su vez está fuertemente correlacionado con el nivel declarado de la propia salud, donde a mayor felicidad personal mejor salud.

\section{A MODO DE SINTESIS}

Los trabajadores informales son mayoritariamente adultos, de ambos sexos, casados, y propietarios de las viviendas en que viven, las que a su vez están emplazadas en las mismas ciudades de trabajo. La mayoría ha trabajado antes formalmente $y$ se 
autodefinen como comerciantes generalmente satisfechos con su trabajo, el que modalmente desempeñan 7 días a la semana. Obtienen aproximadamente $\$ 267.000$ (U\$ 566,8) ${ }^{3}$ mensuales, lo que casi duplica el ingreso mínimo legal en Chile de $\$ 144.000$ a julio de 2007.

Respecto de las causas para desempeñarse en su actual trabajo, el no haber encontrado trabajo formal es mayoritario $(60,1 \%) \mathrm{y}$, si bien la mayoría no cree que ello se deba a discriminación en su contra, un importante porcentaje $(37,9)$ cree que sí lo fue. Aproximadamente la mitad de los trabajadores informales señalan que dejaron voluntariamente un trabajo formal previo para dedicarse al trabajo informal y actualmente la gran mayoría se desempeña exclusivamente en lo informal $(85 \%)$ y sólo un $15 \%$ también en lo formal. Para la inmensa mayoría una ventaja del trabajo informal es la libertad de desempeñarlo los días y por la cantidad de horas que desean $(91 \%)$ recibiendo diariamente dinero por ello.

Los trabajadores informales se reportan muy felices con sus familias en primer lugar y también aunque un poco menos - con su trabajo, moderadamente felices respecto del ocio (o descanso personal), y débilmente felices respecto de sus amigos Así, ellos son personas que valorizan muy positivamente su trabajo, siendo probable que se sitúen en el eje de lo vocacional ó, -como explicación alternativa o complementaria- que el hecho de aquél les permita obtener ingresos superiores al que obtendrían en un trabajo asalariado bajo otras condiciones de realización, sea determinante de su valorización positiva. Así, desde este punto de vista subjetivo, la calidad de su trabajo evaluada por ellos mismos no es mala y aún, si sólo se considera los ingresos, es probablemente mejor que la que podrían obtener como asalariados. Por cierto, desde la perspectiva de lo que es una situación deseable de seguridad y protección sociales, su trabajo es uno precario y de mala calidad.

El B.P. parece compartir más significados comunes con la S.V. que con la felicidad, y estas dos últimas entre sí más que cualquiera de ambas con el B. P. Así, S.V. y felicidad tal como medidas aquí se recubren en gran medida aunque mantienen una importante fracción de separación o independencia, sugiriendo que se trata de constructos diferentes. Sería interesante someter a verificación en futuras investigaciones referidas al trabajo informal qué pesa más en las evaluaciones de la S.L., si el dinero obtenido, o la mayor autonomía relativa de

3 Un dólar equivale a 471 pesos chilenos a enero de 2008. organización laboral y vital comparada con la de un empleo formal. Al tratarse de trabajadores de baja escolaridad la importancia del ingreso sería lo determinante, pero eventualmente también pueden existir aspectos vocacionales y de autonomía que intervengan en su evaluación.

Finalmente, respecto de su salud, los vendedores ambulantes declaran casi tantos días de mala salud física como mental mensualmente $\left(\begin{array}{lll}6 & \text { y } & 5\end{array}\right.$ respectivamente), tres días en que ambas fueron malas, y un día y medio en que debieron abstenerse de trabajar, es decir, normalmente no pueden abstenerse de trabajar no obstante se sientan enfermos.

\section{REFERÊNCIAS}

Argyle, M. (1992). Psicología de la felicidad. Madrid: Alianza Editorial.

Banco Central de Chile (2007). Boletín Mensual, $N^{o}$ 958, Diciembre.

Carty, L. (1993, may). The debate over job quality in the United States: 1970-1990. First Draft, OIT. PREALC.

Csikszentmihalyi, M. (2004). Fluir (flow): Una psicología de la felicidad ( $10^{\mathrm{a}}$ ed.). Barcelona; Kairos.

De Soto, H. (1987). El otro sendero. Santiago; Chile: Centro de Estudios Públicos.

Díaz, J. F. \& Sánchez-López, M. P. (2002). Relaciones entre estilos de personalidad y satisfacción autopercibida en diferentes áreas vitales. Psicothema, 14(1), 100-105.

Diener, E. (1984). Subjective well-being. Psychological Bulletin, 95, 542-575.

Furnham, A., \& Chang, H. (2000). Lay theories of happiness. Journal of Happiness Studies, 1, 227-246.

Hagerty, M., Cummins, R. A., Ferriss, A. L., Land, K. Michalos, A., Peterson, M., Sharpe, A., Sirgy, J., \& Vogel, J. (2001). Quality of life indexes for national policy: Review and agenda for research. Social Indicators Research, 55(1), 1-96.

Hennessy, C., Moriarty, D., Zack, M., Scherr, P., \& Brackbill, R. (1994). Measuring health-related quality of life for public health surveillance. Public Health Reports, 109, 665-672.

Layard, R. (2003). The secrets of happiness. Bajado en 13 de septiembre, 2006 de http://cep.lse.ac.uk/events/ lectures/layard/RL050303.pdf

Market: Latin America (2004). Argentina legitimatizes its informal economy. Market Focus, December.

Lyubomirsky, S., \& Lepper, H. S. (1999). A measure of subjective happiness: Preliminary reliability and construct validation. Social Indicators Research, 2, 137-155.

Moyano Díaz, E. (2004a). Calidad de vida: Felicidad en el trabajo, la familia y las relaciones sociales. [Proyecto de Investigación]. Universidad de Talca, Chile: Dirección de Investigación y Asistencia Técnica-DIAT.

Moyano Díaz, E. (2004b). Las escalas de felicidad y satisfacción vital en estudiantes universitarios. [Documento de Trabajo]. Universidad de Talca: Centro de Psicología Aplicada-CEPA. 
Myers, D. G., \& Diener, E. (1997). The pursuit of happines. Scientific American, Special Issue: The Mind, 7(1), 44-47.

Myers, D. G. (2000). The funds, friends, and faith of happy people. American Psychologist, 55(1), 56-67.

Ocasio, G. (2005, January). The formal problem of the informal economy. Caribbean Business, 33(2), 12.

OIT- Organización Internacional del Trabajo (1999). Trabajo decente. Memoria del Director General. Conferencia Internacional del Trabajo, $87^{\circ}$ Reunión. Ginebra: OIT.

OIT- Organización Internacional del Trabajo (2002). El trabajo decente y la economía informal. [Informe VI. Sexto punto del orden del día]. Conferencia Internacional del Trabajo, $90^{\circ}$ Reunión. Ginebra: OIT.
OIT- Organización Internacional del Trabajo (2003). Panorama laboral 2003 América Latina y el Caribe. Ginebra: OIT.

Seligman, M. (2003). La auténtica felicidad. Barcelona: J. Vergara.

Touraine, A. (1998). Chile: Crecimiento, empleo y el desafí de la justicia social. Ginebra: OIT.

Yunus, M. (2000). Hacia un mundo sin pobreza (7 $7^{\mathrm{a}}$ ed.). Santiago, Chile: Andrés Bello.

Recebido em 20/05/2008

Aceito em 27/07/2008

Endereço para correspondência : Emilio Moyano Díaz. Facultad de Psicología, Universidad de Talca, Av. Lircay, s/n, Talca, Chile.E-mail: emoyano@utalca.cl 